\title{
Clinical characteristics and outcomes of patients with takotsubo syndrome versus spontaneous coronary artery dissection
}

\author{
Victoria L. Cammann ${ }^{1 *}$, Annahita Sarcon ${ }^{2 *}$, Konrad A. Szawan ${ }^{1}$,
} Michael Würdinger ${ }^{1}$, Saif Azam ${ }^{3}$, Jerold Shinbane ${ }^{3}$, Burkhardt Seifert ${ }^{4}$, Jelena R. Ghadri ${ }^{1}$, Jacqueline Saw ${ }^{5 *}$, Christian Templin ${ }^{1 *}$

${ }^{1}$ University Heart Center, Department of Cardiology, University Hospital Zurich, Switzerland

${ }^{2}$ Section of Cardiac Electrophysiology, Department of Medicine,

University of California-San Francisco, CA, United States

${ }^{3}$ University of Southern California, Keck School of Medicine, Los Angeles, CA, United States

${ }^{4}$ Division of Biostatistics, Epidemiology, Biostatistics and Prevention Institute, University of Zurich, Switzerland

${ }^{5}$ Division of Cardiology, Vancouver General Hospital, Vancouver, British Columbia, Canada

\begin{abstract}
Background: Takotsubo syndrome (TTS) and spontaneous coronary artery dissection (SCAD) are now increasingly recognized. Both conditions predominantly affect females; however, the exact pathophysiology remains unclear. Large multi-center databases can help elucidate the underlying mechanism and optimize treatments to improve outcomes by allowing us to compare features and outcomes of patients with TTS and patients with SCAD.

Methods: Takotsubo syndrome patients were enrolled from the International Takotsubo Registry and compared to SCAD patients from the Canadian Spontaneous Coronary Artery Dissection Cohort Study. In total 2098 TTS patients and 750 SCAD patients were included in the present study.

Results: More than $85 \%$ of patients in both groups were females. TTS patients were older compared to SCAD patients. Physical triggers were more common in TTS patients, while emotional triggers and non-identifiable triggering events were more common in SCAD patients. Left ventricular ejection fraction was more impaired in TTS compared to SCAD. TTS patients had more major cardiovascular risk factors, while SCAD patients had a higher rate of migraines and anxiety disorders than TTS patients. Thirty-day mortality was significantly higher in TTS patients, while 30-day stroke rates were comparable between groups.

Conclusions: These findings suggest that women are at higher risk for TTS and SCAD compared to men, which should be considered in the differential diagnosis of those presenting with acute coronary syndrome. Additionally, emotional stressors play a significant role in triggering events particularly in younger women suffering from SCAD. The present findings may help clinicians better differentiate these two entities and aid in the appropriate risk stratification, diagnosis, and management.

Trial registration: ClinicalTrials.gov no. NCT01947621. (Cardiol J 2023; 30, 1: 125-130)

Key words: takotsubo syndrome, spontaneous coronary artery dissection
\end{abstract}

Address for correspondence: Christian Templin, MD, PhD, FESC, Professor of Cardiology, Director Acute Cardiac Care, Director Andreas Grüntzig Heart Catheterization Laboratories, University Hospital Zurich, University Heart Center, Department of Cardiology, Raemistrasse 100, 8091 Zurich, Switzerland, tel: +41 (0) 44255 9585,

e-mail: Christian.Templin@usz.ch

Received: 8.02.2021 Accepted: 7.06.2021 Early publication date: 16.06.2021

*Equally contributed

This article is available in open access under Creative Common Attribution-Non-Commercial-No Derivatives 4.0 International (CC BY-NC-ND 4.0) license, allowing to download articles and share them with others as long as they credit the authors and the publisher, but without permission to change them in any way or use them commercially. 


\section{Introduction}

Advancements in diagnostic techniques, particularly echocardiography and coronary angiography, have led to recognition a novel classification in acute coronary syndromes (ACS), caused by non-obstructive coronary artery disease, coining the term myocardial infarction with non-obstructive coronary arteries (MINOCA). MINOCA encompasses a diverse category of patients with symptoms and positive cardiac biomarkers without obstructive coronary artery disease [1]. Spontaneous coronary artery dissection (SCAD) and takotsubo syndrome (TTS) are increasingly recognized conditions in MINOCA due to heightened awareness.

In 2014 the literature reported $24-35 \%$ of myocardial infarction in women age $<60$ years as a result of SCAD. SCAD has been reported in $1.0-4.0 \%$ of all patients presenting with ACS $[2,3]$. Similarly, $1-4 \%$ of patients presenting with elevated troponin and ACS are diagnosed with TTS [4-6]. SCAD is due to nonatherosclerotic separation of the coronary artery wall and predominantly affects the female population [2]. Similarly, TTS has a predilection for female patients and is probably due to a catecholamine surge causing transient myocardial stunning [7]. The exact pathophysiology of both entities remains unclear. Therefore, this study aimed to compare clinical features and outcomes of TTS patients from the International Takotsubo Registry to SCAD patients from the Canadian Spontaneous Coronary Artery Dissection Cohort Study.

\section{Methods}

\section{Study population}

Takotsubo syndrome patients were enrolled from the International Takotsubo Registry, which is a multicenter study across 35 centers in 11 countries. TTS was diagnosed according to InterTAK Diagnostic Criteria [5]: 1) Transient left ventricular (LV) wall motion abnormality presenting as apical ballooning or midventricular, basal or focal wall motion abnormalities. The wall motion abnormality usually extends beyond a single epicardial coronary artery distribution; however, in rare cases, especially in focal TTS, wall motion abnormality may be limited to a single coronary artery distribution. TTS patients who died during the acute phase, before complete recovery of $\mathrm{LV}$ wall motion, were also eligible for inclusion. 2) A TTS event is typically triggered by emotional, physical or combined stress. However, this is not obligatory. Neurologic disorders or pheochromocytoma may also serve as triggers.
3) The appearance of new electrocardiographic (ECG) abnormalities. Occasionally there may not be any ECG changes. 4) Occurrence of moderate elevations of cardiac biomarkers (troponin and/or creatine kinase). Elevation of B-type natriuretic peptide is common. 5) Coexisting significant coronary artery disease, which is not related to the wall motion abnormality, does not rule out TTS. 6) No evidence of infectious myocarditis.

Clinical characteristics and outcomes of 2098 TTS patients from the InterTAK Registry were compared to a previously published study by Saw et al. [8], which included 750 SCAD patients from the Canadian SCAD cohort study. SCAD was documented by review of coronary angiograms and categorized according to an angiographic classification by Saw et al. [8] in three distinct SCAD types as previously described $[8,9]$. Patients with atherosclerotic disease with diameter $\geq 50 \%$ were not included in the Canadian SCAD cohort study [8].

\section{Statistical analysis}

Continuous variables are presented as means with standard deviations or medians with quartiles, whereas categorial variables are presented as percentages. Continuous variables expressed as medians with quartiles in SCAD were assessed graphically for their distribution in TTS, using histograms and Q-Q plots. Symmetry of the distributions in both groups was assessed visually using distances of the quartiles to the median. An approximately normal distribution of the following variables: height, weight, body mass index, and left ventricular ejection fraction (LVEF) on admission was considered acceptable. They were compared between TTS and SCAD using the Welch test, in which the mean in SCAD was estimated as the median and the standard deviation was estimated as the interquartile range (IQR) divided by 1.349. Categorial variables were compared using Pearson's chi-squared test with Yates' continuity correction. Statistical analyses were performed using $\mathrm{R}$ version 3.5.1 (R Foundation for Statistical Computing, Vienna, Austria).

\section{Results}

A total of 2098 TTS and 750 SCAD patients were included, with main patient characteristics summarized in Table 1 . TTS patients were older $(66.9 \pm 12.8$ years vs. $51.8 \pm 10.2$ years; $\mathrm{p}<$ $<0.001)$. There were no sex differences observed between groups $(90.4 \%$ vs. $88.5 \% ; \mathrm{p}=0.17)$ because both groups comprised predominantly 
Table 1. Characteristics of patients with takotsubo syndrome and spontaneous coronary artery dissection.

\begin{tabular}{|c|c|c|c|}
\hline Characteristic & $\begin{array}{c}\text { TTS } \\
\text { InterTAK Registry } \\
(\mathbf{n}=2098)\end{array}$ & $\begin{array}{c}\text { SCAD } \\
\text { Saw et al. [15] } \\
(n=750)\end{array}$ & $\mathbf{P}$ \\
\hline \multicolumn{4}{|l|}{ Demographics } \\
\hline Female sex - no./total no. (\%) & $1896 / 2098(90.4)$ & $664 / 750(88.5)$ & 0.17 \\
\hline Age [years] & $66.9 \pm 12.8(\mathrm{~N}=2098)$ & $51.8 \pm 10.2(\mathrm{~N}=750)$ & $<0.001$ \\
\hline Height $[\mathrm{cm}]$ & $1.64 \pm 0.7(N=1554)$ & $1.65 \pm 0.08(\mathrm{~N}=750)$ & 0.003 \\
\hline Weight $[\mathrm{kg}]$ & $65.0 \pm 12.6(N=1597)$ & $73.0 \pm 12.6(\mathrm{~N}=750)$ & $<0.001$ \\
\hline \multicolumn{4}{|l|}{ Triggers - no./total no. (\%) } \\
\hline Physical trigger & $758 / 2098(36.1)$ & $216 / 750(28.8)$ & $<0.001$ \\
\hline Emotional trigger & $622 / 2098(29.6)$ & $377 / 750(50.3)$ & $<0.001$ \\
\hline No evident trigger & $570 / 2098(27.2)$ & $252 / 750(33.6)$ & 0.001 \\
\hline \multicolumn{4}{|c|}{ Symptoms on admission - no./total no. (\%) } \\
\hline Chest pain & $1409 / 1894(74.4)$ & $686 / 750(91.5)$ & $<0.001$ \\
\hline Dyspnea & $887 / 1895$ (46.8) & $7 / 750(0.9)$ & $<0.001$ \\
\hline \multicolumn{4}{|l|}{ Troponin - no./total no. (\%) } \\
\hline Elevated troponin & $1407 / 1599(88.0)$ & $732 / 750(97.6)$ & $<0.001$ \\
\hline \multicolumn{4}{|c|}{ ECG on admission - no./total no. (\%) } \\
\hline ST-segment elevation & $794 / 1845(43.0)$ & $223 / 750(29.7)$ & $<0.001$ \\
\hline ST-segment depression & $142 / 1845(7.7)$ & $47 / 750(6.3)$ & 0.24 \\
\hline T-wave inversion & $765 / 1845$ (41.5) & $138 / 750(18.4)$ & $<0.001$ \\
\hline Left bundle branch block & $84 / 1845$ (4.6) & $5 / 750(0.7)$ & $<0.001$ \\
\hline \multicolumn{4}{|l|}{ LVEF on admission } \\
\hline LVEF & $40.0 \pm 10.4(\mathrm{~N}=1851)$ & $55.0 \pm 7.4(\mathrm{~N}=750)$ & $<0.001$ \\
\hline LVEF $<50 \%-$ no./total no. $(\%)$ & $1426 / 1851(77.0)$ & $188 / 734(25.6)$ & $<0.001$ \\
\hline LVEF $<35 \%$ - no./total no. (\%) & $477 / 1851(25.8)$ & 28/734 (3.8) & $<0.001$ \\
\hline \multicolumn{4}{|c|}{ Cardiovascular risk factors - no./total no. (\%) } \\
\hline Hypertension & $1340 / 2042(65.6)$ & $241 / 750(32.1)$ & $<0.001$ \\
\hline Diabetes mellitus & $312 / 2039(15.3)$ & $34 / 750(4.5)$ & $<0.001$ \\
\hline Current smoking & $393 / 1963(20.0)$ & $87 / 750(11.6)$ & $<0.001$ \\
\hline Positive family history of CAD & $349 / 1765$ (19.8) & $285 / 750(38.0)$ & $<0.001$ \\
\hline Hypercholesterolemia & $658 / 2009(32.8)$ & $152 / 750(20.3)$ & $<0.001$ \\
\hline \multicolumn{4}{|l|}{ Medical history - no./total no. (\%) } \\
\hline Migraine & $88 / 1859(4.7)$ & $244 / 750(32.5)$ & $<0.001$ \\
\hline Depression & $351 / 1858(18.9)$ & $146 / 750(19.5)$ & 0.78 \\
\hline Anxiety & $125 / 1858(6.7)$ & $148 / 750(19.7)$ & $<0.001$ \\
\hline Hypothyroidism & $256 / 2024(12.6)$ & $85 / 750(11.3)$ & 0.38 \\
\hline \multicolumn{4}{|c|}{ Medication at discharge - no./total no. (\%) } \\
\hline ACEI or ARB & $1450 / 1832(79.1)$ & $430 / 749(57.4)$ & $<0.001$ \\
\hline Beta-blocker & $1397 / 1832(76.3)$ & $632 / 749(84.4)$ & $<0.001$ \\
\hline Calcium-channel antagonist & $161 / 1832(8.8)$ & $78 / 749(10.4)$ & 0.22 \\
\hline Statin & $932 / 1832(50.9)$ & $413 / 749(55.1)$ & 0.054 \\
\hline ASA & $1186 / 1832(64.7)$ & $702 / 749(93.7)$ & $<0.001$ \\
\hline ADP-receptor antagonist & $210 / 1832(11.5)$ & $505 / 749(67.4)$ & $<0.001$ \\
\hline \multicolumn{4}{|c|}{ Acute cardiac care treatment - no./total no. (\%) } \\
\hline Catecholamine use & 255/2081 (12.3) & $9 / 750(1.2)$ & $<0.001$ \\
\hline Intra-aortic balloon pump & $51 / 2081(2.5)$ & $6 / 750(0.8)$ & 0.009 \\
\hline \multicolumn{4}{|l|}{ Outcomes - no./total no. (\%) } \\
\hline In-hospital death & $93 / 2098(4.4)$ & $1 / 750(0.1)$ & $<0.001$ \\
\hline 30-day-mortality & $93 / 2098(4.4)$ & $1 / 750(0.1)$ & $<0.001$ \\
\hline 30-day Stroke/TIA & 23/2098 (1.1) & $9 / 750(1.2)$ & 0.97 \\
\hline
\end{tabular}

ACEI — angiotensin-converting enzyme inhibitor; ADP — adenosine diphosphate; ARB — angiotensin-receptor blocker; ASA — acetylsalicylic acid; CAD - coronary artery disease; ECG - electrocardiogram; LVEF — left ventricular ejection fraction; TIA - transient ischemic attack; TTS - syndrome takotsubo; SCAD — spontaneous coronary artery dissection 


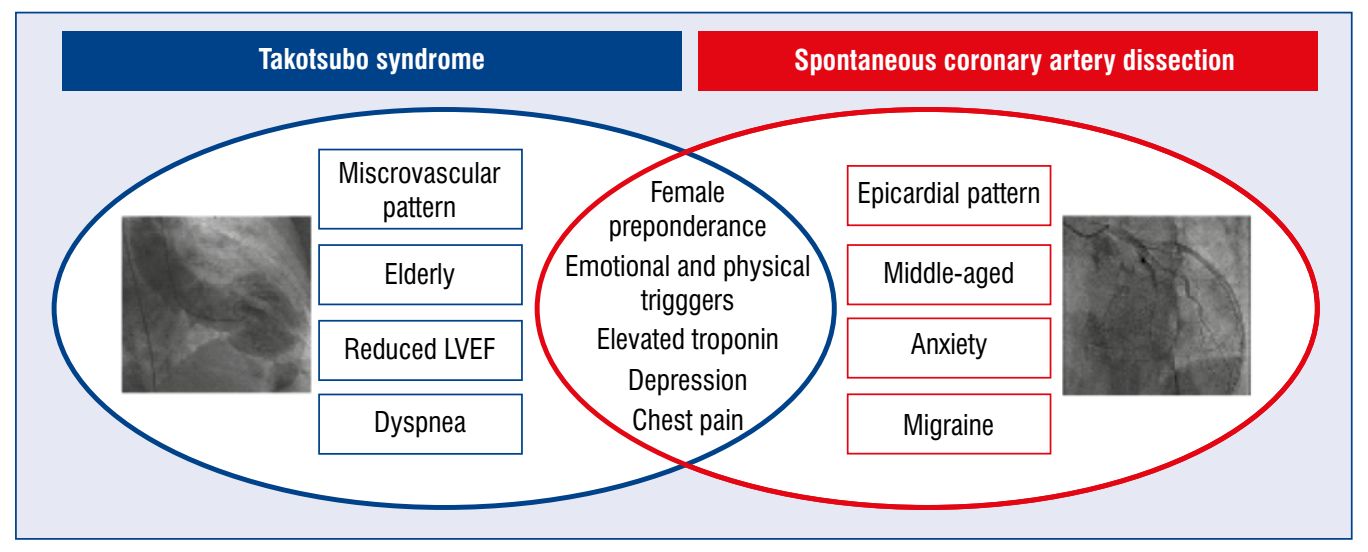

Figure 1. Differences and similarities (intersection) of takotsubo syndrome and spontaneous coronary artery dissection; LVEF — left ventricular ejection fraction.

females. Physical triggers were more common in TTS patients $(36.1 \%$ vs. $28.8 \%$; p $<0.001)$, while emotional triggers $(50.3 \%$ vs. $29.6 \%$; $<0.001)$ or non-identifiable triggering events $(33.6 \%$ vs. $27.2 \% ; \mathrm{p}=0.001$ ) were more common amongst SCAD patients. TTS patients more often had dyspnea $(46.8 \%$ vs. $0.9 \%$; $p<0.001)$ but less frequently presented with chest pain $(74.4 \%$ vs. $91.5 \%$; $\mathrm{p}<0.001)$. ST-segment elevation (43.0\% vs. $29.7 \%$; $\mathrm{p}<0.001)$, T-wave inversion $(41.5 \%$ vs. $18.4 \%$; $\mathrm{p}<0.001)$, and left bundle branch block (4.6\% vs. $0.7 \% ; \mathrm{p}<0.001$ ) were detected more frequently in TTS patients on admission electrocardiogram, but no significant differences in ST-segment depression ( $7.7 \%$ vs. $6.3 \% ; p=0.24)$ were reported. LVEF was more impaired in TTS patients $(40.0 \pm 10.4 \%$ vs. $55.0 \pm 7.4 \%$; $\mathrm{p}<0.001$ ). TTS patients had more cardiovascular risk factors including hypertension, current smoking, diabetes mellitus, family history, or hypercholesterolemia. Of note, SCAD patients had a higher rate of migraines $(32.5 \%$ vs. $4.7 \%$; $<<0.001)$ and anxiety disorders (19.7\% vs. $6.7 \%$; $\mathrm{p}<0.001)$.

At discharge, TTS patients were more likely to be prescribed angiotensin-converting enzyme inhibitors or angiotensin-receptor blockers $(79.1 \%$ vs. $57.4 \%$; $\mathrm{p}<0.001$ ), while patients with SCAD were discharged more often on beta-blockers ( $84.4 \%$ vs. $76.3 \%$; $p<0.001)$, acetylsalicylic acid $(93.7 \%$ vs. $64.7 \%$; $<<0.001)$, and adenosine diphosphate receptor antagonists $(67.4 \%$ vs. $11.5 \%$; $\mathrm{p}<0.001$ ).

Takotsubo syndrome patients required more acute cardiac care treatment including catecholamine administration $(12.3 \%$ vs. $1.2 \% ; \mathrm{p}<0.001)$ and hemodynamic support with intra-aortic balloon pump ( $2.5 \%$ vs. $0.8 \%$; $p=0.009)$. Thirty-day mortality was significantly higher in TTS patients ( $4.4 \%$ vs. $0.1 \% ; \mathrm{p}<0.001)$, while 30 -day stroke rates were comparable between the groups $(1.1 \%$ vs. $1.2 \% ; \mathrm{p}=0.97)$.

\section{Discussion}

Our observational study reveals noteworthy findings in these unique patient populations. While the majority of patients were females, TTS patients were substantially older with more cardiovascular risk factors. However, interestingly, the SCAD population had a higher prevalence of anxiety and migraines. This may be age related because multiple studies have shown that the prevalence of migraines and anxiety disorders among women decreases after menopause [10-13]. It is also surprising, given the traditional description of TTS as "broken heart syndrome", that emotional triggers were identified more in the SCAD population. However, compared to ACS patients, the TTS group had a higher prevalence of psychiatric disorders, including anxiety and depression [14]. These observations are hypothesis generating but offer useful insights into risk stratifying female patients with acute chest pain. Importantly, while both SCAD and TTS are associated with predisposing "stressful triggers", the prevalence and type of triggers is different between the two groups. Physical triggers in SCAD were often exertional, while TTS physical triggers were often related to alterations in the physiological milieu such as intracranial hemorrhage or asthma exacerbation [14-16]. These distinctions may help explain the difference in 30-day mortality rates observed in our study with higher mortality rates in the TTS group (Fig. 1). 
Takotsubo syndrome in the form of typical LV apical ballooning can co-exist in SCAD patients, even without a "wrap-around" course of the left anterior descending artery [13]. Therefore, distinguishing between the two conditions is important given the different management strategies. Treatment of TTS is often conservative; however, cardiopulmonary support including mechanical device therapy and ventilator support may be required in patients with severe cardiogenic shock $[14,17,18]$. Similarly, SCAD patients are typically managed conservatively in the absence of clinical deterioration [19]. In cases involving left main or extensive proximal vessel dissection, percutaneous coronary intervention or coronary artery bypass graft is required [19]. SCAD lesions usually heal spontaneously, and LVEF is either preserved or recovers shortly after [15], similarly to LVEF recovery in TTS.

While this study illustrates observations from a non-randomized population and has its limitations, it includes a large number of patients. This offers remarkable clinical insight into acute cardiovascular conditions that particularly affect women. While postmenopausal women are predominately affected by TTS, young to middle-aged women suffer from SCAD. Therefore, in women presenting with ACS, SCAD and TTS should be considered as the differential diagnosis with special attention to characteristics that harbinger poor clinical outcomes prompting vigorous clinical vigilance. Emotional and physical stressors are a common denominator, and perhaps a paradigm shift in risk factor modification should be implanted upon discharge. Approximately $29 \%$ of SCAD and $2-10 \%$ of TTS cases have the risk of recurrence [19-21]. These statistics might underestimate the true number of cases because these conditions continue to remain misdiagnosed or underdiagnosed. Perhaps in addition to medical therapy, unconventional therapeutic modalities including stress reduction, a multidisciplinary approach, and providing psychosocial support might also be beneficial. Additionally, given the overlapping characteristics in these two populations and the possibility of SCAD-TTS co-existence [22], prompt diagnostics such as angiography and repeated imaging can avoid incorrect management and undesirable outcomes.

\section{Conclusions}

The present findings may help clinicians better differentiate TTS and SCAD entities and aid in the appropriate risk stratification, diagnosis, and (probably) management.

\section{Funding}

Christian Templin has been supported by the H.H. Sheikh Khalifa bin Hamad Al-Thani Research Program and the Swiss Heart Foundation. The InterTAK Registry is supported by the Biss Davies Charitable Trust.

Conflict of interest: Doctor Christian Templin reports receiving consulting fees from Biotronik, Microport and Schnell Medical; lecture fees from Novartis; and serving on advisory boards from Amgen. All other authors declare no conflict of interest.

\section{References}

1. Agewall S, Beltrame JF, Reynolds HR, et al. ESC working group position paper on myocardial infarction with non-obstructive coronary arteries. Eur Heart J. 2017; 38(3): 143-153, doi: 10.1093/ eurheartj/ehw149, indexed in Pubmed: 28158518.

2. Hayes SN, Kim ESH, Saw J, et al. Spontaneous coronary artery dissection: current state of the science: a Scientific Statement from the American Heart Association. Circulation. 2018; 137(19): e523-e557, doi: 10.1161/CIR.0000000000000564, indexed in Pubmed: 29472380.

3. Mahmoud AN, Taduru SS, Mentias A, et al. Trends of incidence, clinical presentation, and in-hospital mortality among women with acute myocardial infarction with or without spontaneous coronary artery dissection: a population-based analysis. JACC Cardiovasc Interv. 2018; 11(1): 80-90, doi: 10.1016/j. jcin.2017.08.016, indexed in Pubmed: 29248409.

4. Gianni M, Dentali F, Grandi AM, et al. Apical ballooning syndrome or takotsubo cardiomyopathy: a systematic review. Eur Heart J. 2006; 27(13): 1523-1529, doi: 10.1093/eurheartj/ehl032, indexed in Pubmed: 16720686.

5. Ghadri JR, Wittstein IS, Prasad A, et al. International expert consensus document on takotsubo syndrome (part I): clinical characteristics, diagnostic criteria, and pathophysiology. Eur Heart J. 2018; 39(22): 2032-2046, doi: 10.1093/eurheartj/ehy076, indexed in Pubmed: 29850871.

6. Ghadri JR, Cammann VL, Jurisic S, et al. A novel clinical score (InterTAK Diagnostic Score) to differentiate takotsubo syndrome from acute coronary syndrome: results from the International Takotsubo Registry. Eur J Heart Fail. 2017; 19(8): 1036-1042, doi: 10.1002/ejhf.683, indexed in Pubmed: 27928880.

7. Lyon AR, Rees PSC, Prasad S, et al. Stress (Takotsubo) cardiomyopathy--a novel pathophysiological hypothesis to explain catecholamine-induced acute myocardial stunning. Nat Clin Pract Cardiovasc Med. 2008; 5(1): 22-29, doi: 10.1038/ncpcardio1066, indexed in Pubmed: 18094670.

8. Saw J, Humphries K, Aymong E, et al. Spontaneous coronary artery dissection: clinical outcomes and risk of recurrence. J Am Coll Cardiol. 2017; 70(9): 1148-1158, doi: 10.1016/j. jacc.2017.06.053, indexed in Pubmed: 28838364.

9. Saw J, et al. Coronary angiogram classification of spontaneous coronary artery dissection. Catheter Cardiovasc Interv. 2014; 84(7): 1115-1122, doi: 10.1002/ccd.25293, indexed in Pubmed: 24227590 .

10. Kelman L. Migraine changes with age: IMPACT on migraine classification. Headache. 2006; 46(7): 1161-1171, doi: 10.1111/j.15264610.2006.00444.x, indexed in Pubmed: 16866720. 
11. Freitag FG. Why do migraines often decrease as we age? Curr Pain Headache Rep. 2013; 17(10): 366, doi: 10.1007/s11916-0130366-3, indexed in Pubmed: 23996722.

12. Jorm AF. Does old age reduce the risk of anxiety and depression? A review of epidemiological studies across the adult life span. Psychol Med. 2000; 30(1): 11-22, doi: 10.1017/ s0033291799001452, indexed in Pubmed: 10722172.

13. Lenze EJ, Wetherell JL. A lifespan view of anxiety disorders. Dialogues Clin Neurosci. 2011; 13(4): 381-399, indexed in Pubmed: 22275845.

14. Templin C, Ghadri JR, Diekmann J, et al. Clinical Features and Outcomes of Takotsubo (Stress) Cardiomyopathy. N Engl J Med. 2015; 373(10): 929-938, doi: 10.1056/NEJMoa1406761, indexed in Pubmed: 26332547.

15. Saw J, Starovoytov A, Humphries K, et al. Canadian spontaneous coronary artery dissection cohort study: in-hospital and 30-day outcomes. Eur Heart J. 2019; 40(15): 1188-1197, doi: 10.1093/ eurheartj/ehz007, indexed in Pubmed: 30698711.

16. Gili S, Cammann VL, Schlossbauer SA, et al. Cardiac arrest in takotsubo syndrome: results from the InterTAK Registry. Eur Heart J. 2019; 40(26): 2142-2151, doi: 10.1093/eurheartj/ehz170, indexed in Pubmed: 31098611.

17. Di Viece D, Citro R, Cammann VL. Outcomes Associated With Cardiogenic Shock in Takotsubo Syndrome: Results From the
International Takotsubo Registry. Circulation. 2019; 139(3): 413-415, doi: 10.1161/CIRCULATIONAHA.118.036164, indexed in Pubmed: 30586690.

18. Rashed A, Won S, Saad M, et al. Use of the Impella 2.5 left ventricular assist device in a patient with cardiogenic shock secondary to takotsubo cardiomyopathy. BMJ Case Rep. 2015; 2015, doi: 10.1136/bcr-2014-208354, indexed in Pubmed: 25953675.

19. Tweet MS, Hayes SN, Pitta SR, et al. Clinical features, management, and prognosis of spontaneous coronary artery dissection. Circulation. 2012; 126(5): 579-588, doi: 10.1161/CIRCULATIONAHA.112.105718, indexed in Pubmed: 22800851.

20. Kato K, Di Vece D, Cammann VL, et al. Takotsubo recurrence: morphological types and triggers and identification of risk factors. J Am Coll Cardiol. 2019; 73(8): 982-984, doi: 10.1016/j. jacc.2018.12.033, indexed in Pubmed: 30819368.

21. Angelini P. Recurrent takotsubo cardiomyopathy: an opportunity to clarify causation and prognosis. Tex Heart Inst J. 2018; 45(4): 252-253, doi: 10.14503/THIJ-18-6778, indexed in Pubmed: 30374240 .

22. Y-Hassan S, Böhm F. The causal link between spontaneous coronary artery dissection and takotsubo syndrome: A case presented with both conditions. Int J Cardiol. 2016; 203: 828-831, doi: 10.1016/j.ijcard.2015.11.047, indexed in Pubmed: 26599743. 